\title{
Human prion diseases: from antibody screening to a standardized fast immunodiagnosis using automation
}

\author{
Nicolas Privat ${ }^{1,2}$, Isabelle Laffont-Proust ${ }^{1,2}$, Baptiste A Faucheux ${ }^{1,2,3}$, Véronique \\ Sazdovitch $^{1,2}$, Yveline Frobert ${ }^{4}$, Jean-Louis Laplanche ${ }^{5}$, Jacques Grassi ${ }^{4}$, \\ Jean-Jacques Hauw ${ }^{2,3}$ and Stéphane Haïk ${ }^{1,2,3}$
}

${ }^{1}$ INSERM, Avenir Team-Human Prion Diseases, Paris, France; ${ }^{2} I n V S$, Centre national de référence des Agents Transmissibles Non Conventionnels, Paris, France; ${ }^{3}$ AP-HP, R Escourolle Neuropathology Laboratory, Salpêtrière Hospital, Paris, France; ${ }^{4} C E A$, Service de Pharmacologie et d'Immunologie, CEA/Saclay, Gif-sur-Yvette Cedex, France and ${ }^{5}$ AP-HP, Central Laboratory of Biochemistry, Lariboisiere Hospital, Paris, France

\begin{abstract}
Demonstration of pathological prion protein accumulation in the central nervous system is required to establish the diagnosis of transmissible subacute encephalopathies. In humans, this is frequently achieved using prion protein immunohistochemistry in paraffin-embedded tissue, a technique that requires multiple epitope retrieval and denaturing pretreatments. In addition to being time-consuming, this procedure induces tissue alterations that preclude accurate morphological examination. The aim of this study was to simplify prion protein immunohistochemistry procedure in human tissue, together with increased sensitivity and specificity. We screened a panel of 50 monoclonal antibodies produced using various immunogens (human and ovine recombinant prion protein, prion protein peptides, denatured scrapie-associated fibrils from $263 \mathrm{~K}$-infected Syrian hamsters) and directed against different epitopes along the human prion protein sequence. A panel of different forms of genetic, infectious and sporadic transmissible subacute encephalopathies was assessed. The monoclonal 12F10 antibody provided a high specificity and fast immunodiagnosis with very limited denaturing pretreatments. A standardized and reliable fast immunostaining procedure was established using an automated diagnostic system (Nexes, Ventana Medical Systems) and allowed prion protein detection in the central nervous system and in tonsil biopsies. It was evaluated in a series of 300 patients with a suspected diagnosis of transmissible subacute encephalopathies and showed high sensitivity and specificity.
\end{abstract}

Modern Pathology (2008) 21, 140-149; doi:10.1038/modpathol.3800994; published online 14 December 2007

Keywords: prion; Creutzfeldt-Jakob disease; diagnosis

The accumulation of a specific protease-resistant isoform $\left(\mathrm{PrP}^{\mathrm{sc}}\right)$ of the host-encoded prion protein $\left(\mathrm{PrP}^{\mathrm{c}}\right)$ characterizes human transmissible spongiform encephalopathies. Identifying $\mathrm{PrP}^{\mathrm{sc}}$ in the central nervous tissue of suspected patients is a critical step for the diagnosis of definite human transmissible subacute encephalopathies. ${ }^{1,2}$ This is frequently achieved using PrP immunohistochemistry on formalin-fixed tissues.

Retrieval of antigens and denaturing pretreatments is required for detecting $\operatorname{PrP}^{\mathrm{sc}}$ by immunohistochemical procedures in sections of formalin-fixed and paraffin-embedded tissues. Formic acid ${ }^{3}$ and enzy-

Correspondence: N Privat, Department of Neuropathology, INSERM, Avenir Team-Human Prion Diseases-IFR70, Hôpital Pitié-Salpêtrière, 47 Bd de l'hôpital, Paris 75013, France.

E-mail: nicolas.privat@chups.jussieu.fr

Received 29 May 2007; revised 21 September 2007; accepted 01 October 2007; published online 14 December 2007 matic digestion with proteinase $\mathrm{K}^{4}$ or pepsin ${ }^{5}$ have been used at first. Denaturing treatments using guanidine thiocyanate ${ }^{6}$ and heating by hydrolytic autoclaving $^{7}$ or microwave irradiation ${ }^{8}$ and hydrated autoclaving $^{9}$ have also been developed. Various combinations of these methods have been successfully tested. ${ }^{10,11}$ Two multicentric, prospective studies have assessed several antibodies, including the commercially available and widely used $3 \mathrm{~F} 4^{12}$ antibody in combination with enhancement procedures. Regardless of the antibody in use, they have led to the same reliable standardized pretreatment protocol for $\mathrm{PrP}^{\mathrm{sc}}$ immunohistochemistry: hydrated autoclaving $\left(121^{\circ} \mathrm{C}\right.$ for $\left.10 \mathrm{~min}\right)$, followed by formic acid (96\% for $5 \mathrm{~min})$ and guanidine thiocyanate $\left(4 \mathrm{M}, 4^{\circ} \mathrm{C}\right.$ for $\left.2 \mathrm{~h}\right){ }^{13}$ However, the most sensitive procedure needs an additional antigen-retrieving treatment (ie, proteinase $\mathrm{K}$ digestion), ${ }^{14}$ and these complex pretreatments are time-consuming and induce tissue alterations that preclude fine morphological analysis. 
Using a large panel of anti-PrP monoclonal antibodies produced with various immunogens, we have developed a fast, reliable and automated procedure for $\mathrm{PrP}^{\mathrm{sc}}$ immunohistochemistry in human samples, which enables (1) a high sensitivity and specificity for the diagnosis of human transmissible subacute encephalopathies and (2) a fine preservation of morphological information thanks to a simplified antigen-retrieving procedure.

\section{Materials and methods}

\section{Antibody Screening Using a Four-Step Strategy}

Step 1: Screening of 50 antibodies on four patients using a combination of four pretreatments

Our strategy was to study a panel of 50 monoclonal antibodies on four patients using a combination of four pretreatments. Four dilutions of primary antibodies $(1 / 100,1 / 500,1 / 1000,1 / 2000)$ were tested. The staining was compared to that obtained using the 3F4 antibody (Figure 1, Tables 1 and 2).

Step 2: Reducing the pretreatments in the six selected antibodies

The highly efficient antibodies identified in step 1 were used in different simplified pretreatment procedures.
Step 3: Automation of the procedure using the six selected antibodies on 26 patients

These antibodies were checked for their efficiency at $37^{\circ} \mathrm{C}$ using an automated diagnostic system for immunohistochemistry (Nexes, Ventana Medical Systems, Illkirch, France) on 26 patients. The results were compared to those obtained with $3 \mathrm{~F} 4$ antibody using the manual and four pretreatment procedures. The highest sensitivity was obtained with the $12 \mathrm{~F} 10$ antibody.

Step 4: Validation of the automated procedure using an additional series of 25 sporadic CreutzfeldtJakob disease patients

Together with the 26 patients used in step 3 , sections from a total of 51 patients were used to compare the $12 \mathrm{~F} 10$ automated procedure to the manual procedure, with the reference $3 \mathrm{~F} 4$ antibody.

\section{Patients and Samples}

The panel of 51 patients, which included 46 patients with neuropathologically confirmed transmissible subacute encephalopathies, is described in Table 1. Paraffin-embedded blocks of $10 \%$ formalinfixed brains were sampled from the frontal isocortex and the cerebellar vermis. Serial sections cut at $7 \mu \mathrm{m}$ on superfrost-plus slides were dried at $56^{\circ} \mathrm{C}$ for $24 \mathrm{~h}$ before immunohistochemical procedures.

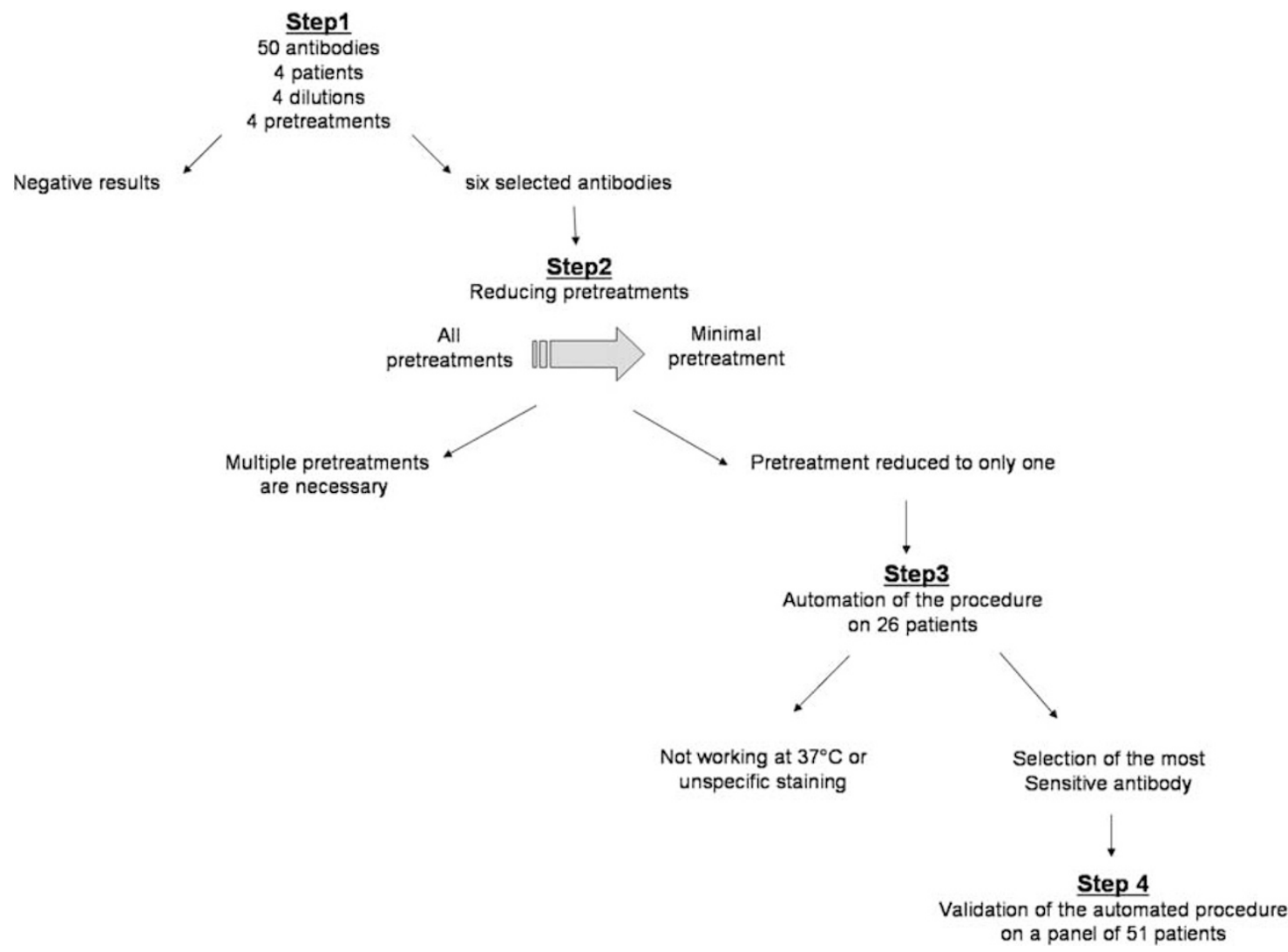

Figure 1 Strategy used for antibody screening. 
Table 1 Patient characteristics

\begin{tabular}{lll}
\hline $\begin{array}{l}\text { Step 1 and step } \\
2(4 \text { patients) }\end{array}$ & Step 3 (26 patients) & Step 4 (51 patients) \\
\hline sCJD MM1 $(n=1)$ & sCJD MM1 $(n=3)$ & sCJD MM1 $(n=20)$ \\
sCJD MV1 $(n=1)$ & sCJD MV1 $(n=3)$ & sCJD MV1 $(n=8)$ \\
sCJD VV2 $(n=1)$ & sCJD MV2 $(n=3)$ & sCJD MV2 $(n=3)$ \\
AD $(n=1)$ & sCJD VV2 $(n=3)$ & sCJD VV2 $(n=6)$ \\
& vCJD $(n=3)$ & vCJD $(n=3)$ \\
& GSS P102L $(n=1)$ & GSS P102L $(n=1)$ \\
& FFI $(n=1)$ & FFI $(n=1)$ \\
& fCJD E200K $(n=1)$ & fCJD E200K $(n=1)$ \\
& iCJD $(n=3)$ & iCJD $(n=3)$ \\
& AD $(n=3)$ & AD $(n=3)$ \\
& NND $(n=2)$ & NND $(n=2)$
\end{tabular}

AD, Alzheimer disease patient; fCJD, inherited Creutzfeldt-Jakob disease with the E200K mutation; FFI, familial fatal insomnia with the D178N-129M mutation; GSS, Gerstmann-Sträussler-Scheinker syndrome with the P102L mutation; iCJD, iatrogenic CJD after extractive growth hormone treatment; MM1, methionine homozygosity at codon 129 of PRNP and a PrP ${ }^{\mathrm{sc}}$ type 1; MV1, methionine/valine heterozygosity at codon 129 and a $\operatorname{PrP}^{\mathrm{sc}}$ type 1; MV2, methionine/valine heterozygosity at codon 129 and a $\operatorname{PrP}^{\mathrm{sc}}$ type $2 \mathrm{~A}$; NND, patients who died from non-neurological diseases; sCJD, sporadic CreutzfeldtJakob disease; vCJD, variant Creutzfeldt-Jakob disease; VV2, valine homozygosity at the codon 129 and a $\operatorname{PrP}^{\mathrm{sc}}$ type $2 \mathrm{~A}$.

\section{Immunohistochemical Procedures}

\section{Pretreatments}

A combination of four pretreatments was used: hydrated autoclaving in distilled water $(25 \mathrm{~min})$, formic acid ( $5 \mathrm{~min}, 99 \%, 24^{\circ} \mathrm{C}$ ), guanidine thiocyanate $\left(2 \mathrm{~h}, 4 \mathrm{M}, 4^{\circ} \mathrm{C}\right)$, and proteinase $\mathrm{K}(8 \mathrm{~min}$, $\left.10 \mu \mathrm{g} / \mathrm{ml}, 24^{\circ} \mathrm{C}\right)$.

\section{Anti-PrP antibodies}

Antibodies were produced in $\mathrm{PrP}^{0 / 0}$ mice using different immunogens (human and ovine recombinant PrP, PrP peptides, denatured scrapie-associated fibrils from 263K-infected Syrian hamsters), and have been described elsewhere. ${ }^{15,16}$ They recognized different epitopes along the PrP sequence (Table 2). Monoclonal antibodies were purified using either caprylic acid precipitation ${ }^{17}$ or protein A affinity chromatography. Epitope mapping for identifying linear epitopes possibly recognized by monoclonal antibodies was performed as previously described..$^{16}$ 3F4 antibody (ref. 9620) was produced and commercialized by Signet Laboratories (MA, USA).

\section{Manual procedure}

After a saturation step using $10 \%$ goat serum for $30 \mathrm{~min}$, a $3 \% \mathrm{H}_{2} \mathrm{O}_{2}$ blockade of endogenous peroxidases and different combinations of pretreatments, the primary antibody was applied overnight at room temperature. A standard streptavidin-biotin peroxidase kit (ref. K5001) with diaminobenzidine substrate (Dako, Trappes, France) was used for single immunolabelling according to the manufacturer's instructions. Sections were counterstained with haematoxylin, dehydrated and mounted.
Table 2 Antibodies screened

\begin{tabular}{|c|c|c|c|}
\hline Antibody & Isotype & Epitope & Immunogen \\
\hline $3 F 4$ & $\operatorname{IgG} 2 \mathrm{a}$ & $109-112$ & $\mathrm{PrP}^{\mathrm{sc}}$ hamster $27-30$ \\
\hline Pri-101 & IgG2a & $23-33$ & Human $\operatorname{PrP}^{\mathrm{c}}$, peptide $23-33$ \\
\hline Pri-102 & IgG2a & $23-33$ & Human $\operatorname{PrP}^{\mathrm{c}}$, peptide $23-33$ \\
\hline Pri-103 & IgG2bk & $23-33$ & Human PrPc , peptide 23-33 \\
\hline Pri-108 & IgG1 & $23-33$ & Human PrPc, peptide 23-33 \\
\hline Pri-151 & IgG1 & $23-33$ & Human $\operatorname{PrP}^{\mathrm{c}}$, peptide $23-33$ \\
\hline Pri-152 & IgG1k & $23-33$ & Human $\operatorname{PrP}^{\mathrm{c}}$, peptide 23-33 \\
\hline Pri-153 & IgG1 & $23-33$ & Human $\operatorname{PrP}^{\mathrm{c}}$, peptide $23-33$ \\
\hline Pri-154 & IgG1k & $23-33$ & Human PrPc , peptide 23-33 \\
\hline Pri-157 & IgG2a & $23-33$ & Human $\operatorname{PrP}^{\mathrm{c}}$, peptide $23-33$ \\
\hline Pri-303 & IgG2ak & $106-126$ & Human $\operatorname{PrP}^{\mathrm{c}}$, peptide $106-126$ \\
\hline Pri-304 & IgG1k & $111-117^{*}$ & Human $\operatorname{PrP}^{\mathrm{c}}$, peptide $106-126$ \\
\hline Pri-305 & IgG2ak & $106-126$ & Human $\mathrm{PrP}^{\mathrm{c}}$, peptide 106-126 \\
\hline Pri-307 & IgG2ak & $106-126$ & Human $\operatorname{PrP}^{\mathrm{c}}$, peptide $106-126$ \\
\hline Pri-308 & IgG1k & $111-118^{*}$ & Human $\mathrm{PrP}^{\mathrm{c}}$, peptide $106-126$ \\
\hline Pri-310 & IgG1k & $106-126$ & Human $\operatorname{PrP}^{\mathrm{c}}$, peptide $106-126$ \\
\hline Pri-313 & IgG1k & $106-126$ & Human $\operatorname{PrP}^{\mathrm{c}}$, peptide $106-126$ \\
\hline Pri-622 & IgG2ak & 161-179 & Human $\mathrm{PrP}^{\mathrm{c}}$, peptide $161-179$ \\
\hline Pri-901 & IgG1 & $213-233$ & Human $\operatorname{PrP}^{\mathrm{c}}$, peptide $213-233$ \\
\hline Pri-904 & IgA & $213-233$ & Human $\mathrm{PrP}^{\mathrm{c}}$, peptide $213-233$ \\
\hline Pri-907 & IgG1 & $213-233$ & Human $\operatorname{PrP}^{\mathrm{c}}$, peptide $213-233$ \\
\hline Pri-908 & IgG1 & $213-233$ & Human $\mathrm{PrP}^{\mathrm{c}}$, peptide $213-233$ \\
\hline Pri-909 & IgG1 & $213-233$ & Human $\mathrm{PrP}^{\mathrm{c}}$, peptide $213-233$ \\
\hline Pri-917 & IgG1 & $216-221^{*}$ & Human $\operatorname{PrP}^{\mathrm{c}}$, peptide $213-233$ \\
\hline 14D3 & IgG1k & $37-53$ & Human recombinant PrP \\
\hline $4 \mathrm{~F} 2$ & IgG2bk & $59-\varepsilon$ & Human recombinant PrP \\
\hline 3B5 & IgG2ak & $59-89 *$ & Human recombinant PrP \\
\hline $8 \mathrm{G} 8$ & IgG2ak & $97-102 *$ & Human recombinant PrP \\
\hline $12 \mathrm{~F} 10$ & IgG2ak & $144-152^{*}$ & Human recombinant $\operatorname{PrP}$ \\
\hline 11C6 & IgG2ak & $\mathrm{D} / \mathrm{U}$ & Human recombinant PrP \\
\hline SAF15 & IgG3k & 79-92 & Denatured $\mathrm{PrP}^{\mathrm{sc}} 263 \mathrm{~K}$ \\
\hline SAF31 & IgG2bk & $79-92$ & Denatured $\mathrm{PrP}^{\mathrm{sc}} 263 \mathrm{~K}$ \\
\hline SAF32 & IgG2bk & 79-92 & Denatured $\mathrm{PrP}^{\mathrm{sc}} 263 \mathrm{~K}$ \\
\hline SAF33 & IgG2bk & $79-92$ & Denatured $\mathrm{PrP}^{\mathrm{sc}} 263 \mathrm{~K}$ \\
\hline SAF34 & IgG2ak & $79-92$ & Denatured $\mathrm{PrP}^{\mathrm{sc}} 263 \mathrm{~K}$ \\
\hline SAF35 & IgG2bk & 79-92 & Denatured $\mathrm{PrP}^{\mathrm{sc}} 263 \mathrm{~K}$ \\
\hline SAF37 & IgG2bk & $79-92$ & Denatured $\operatorname{PrP}^{\mathrm{sc}} 263 \mathrm{~K}$ \\
\hline SAF53 & IgG2ak & $143-153^{*}$ & Denatured $\operatorname{PrP}^{\mathrm{sc}} 263 \mathrm{~K}$ \\
\hline SAF54 & IgG2bk & $157-161^{*}$ & Denatured $\operatorname{PrP}^{\mathrm{sc}} 263 \mathrm{~K}$ \\
\hline SAF60 & IgG2bk & $157-161^{*}$ & Denatured $\mathrm{PrP}^{\mathrm{sc}} 263 \mathrm{~K}$ \\
\hline SAF61 & IgG2ak & $142-153^{*}$ & Denatured PrPsc $263 \mathrm{~K}$ \\
\hline SAF66 & IgG2a & $142-160^{*}$ & Denatured $\mathrm{PrP}^{\mathrm{sc}} 263 \mathrm{~K}$ \\
\hline SAF69 & IgG2bk & $157-161^{*}$ & Denatured $\operatorname{PrP}^{\mathrm{sc}} 263 \mathrm{~K}$ \\
\hline SAF70 & IgG2bk & $156-162^{*}$ & Denatured $\operatorname{PrP}^{\mathrm{sc}} 263 \mathrm{~K}$ \\
\hline SAF75 & IgG2ak & $144-149^{*}$ & Denatured $\mathrm{PrP}^{\mathrm{sc}} 263 \mathrm{~K}$ \\
\hline SAF76 & IgG2ak & $144-149^{*}$ & Denatured $\operatorname{PrP}^{\mathrm{sc}} 263 \mathrm{~K}$ \\
\hline SAF83 & IgG1k & $\mathrm{D} / \mathrm{U}$ & Denatured PrP $P^{\mathrm{sc}} 263 \mathrm{~K}$ \\
\hline SAF84 & IgG2bk & $161-170^{*}$ & Denatured PrPsc $263 \mathrm{~K}$ \\
\hline BAR224 & IgG2a & $141-151$ & Ovine recombinant $\operatorname{PrP}$ \\
\hline BAR226 & IgG2a & $\mathrm{D} / \mathrm{U}$ & Ovine recombinant $\operatorname{PrP}$ \\
\hline BAR233 & IgG2a & $141-152 *$ & Ovine recombinant PrP \\
\hline
\end{tabular}

$\mathrm{D} / \mathrm{U}$, discontinuous/unidentified; $\mathrm{PrP}^{\mathrm{c}}$, host-encoded prion protein; $\mathrm{PrP}^{\mathrm{sc}}$, protease-resistant isoform of prion protein.

*Antibodies recognizing a linear epitope determined by a pepscan method (see Materials and methods).

\section{Automated procedure}

A saturation step using 3\% BSA for $20 \mathrm{~min}$ and hydrated autoclaving pretreatment was applied. The NexES IHC automated diagnostic system for immunohistochemistry and the iVIEW DAB Paraffin kit (ref. 760-091) were used according to the manufacturer's instructions (Ventana Medical Systems). The blocking of endogenous peroxidases, primary antibody incubation (32 min), DAB/copper 
Table 3 Search for the simplified pretreatments for each optimal adjusted dilution (manual procedures)

\begin{tabular}{|c|c|c|c|c|c|c|c|c|c|}
\hline \multirow[t]{2}{*}{ Antibody } & \multirow[t]{2}{*}{ Dilution $(\mu g / m l)$} & \multicolumn{8}{|c|}{ Different pretreatment procedures } \\
\hline & & None & $H A$ & $F A$ & $P K$ & $H A+F A$ & $H A+G T$ & $H A+F A+G T$ & $H A+F A+G T+P K$ \\
\hline $3 \mathrm{~F} 4$ & $1 / 500(2.00)$ & - & - & - & - & - & - & + & ++ \\
\hline $12 \mathrm{~F} 10$ & 1/2000 (0.85) & + & ++ & + & + & ++ & ++ & ++ & ++ \\
\hline $4 \mathrm{~F} 2$ & $1 / 500(2.00)$ & - & ++ & - & - & ++ & ++ & ++ & ++ \\
\hline 3B5 & $1 / 2000(0.95)$ & + & ++ & + & + & ++ & ++ & ++ & ++ \\
\hline SAF54 & $1 / 500(0.80)$ & + & ++ & + & + & ++ & ++ & ++ & ++ \\
\hline SAF34 & $1 / 1000(1.10)$ & + & ++ & - & + & ++ & ++ & ++ & ++ \\
\hline BAR233 & 1/500 (2.00) & - & ++ & - & - & ++ & ++ & ++ & ++ \\
\hline
\end{tabular}

FA, formic acid; GT, guanidine thiocyanate; HA, hydrated autoclaving; PK, proteinase K.

The level of positive immunostaining rated on a three-grade scale: - , no staining; +, positive staining; ++, strong positive staining.

revelation and section counterstaining with haematoxylin were performed at $37^{\circ} \mathrm{C}$ working temperature. Slides were dehydrated and mounted.

\section{PrP Patterns}

For assessing the pattern of immunolabelling with anti-PrP antibodies on brain samples, we followed criteria derived from those used previously. ${ }^{3,10,13,18-20}$ Six types of labelling were distinguished: synaptic labelling (diffuse staining), granular deposits (small scattered immunolabelled spots less than $5 \mu \mathrm{m}$ wide), focal deposits (large, 5-50 $\mu \mathrm{m}$ wide, nonamyloid rounded positive blots), vacuolar deposits (surrounding vacuoles of spongiform change), morula-type deposits (focal, non-amyloid deposits surrounded by vacuoles of spongiform change) and kuru-type amyloid plaques, $10-50 \mu \mathrm{m}$ wide (plaques were also identified on Congo red and periodic acid Schiff-stained sections). ${ }^{14,21}$ We did not consider other types of labelling as significant, such as that of isolated neurons or astrocytes. PrP immunostaining was considered as positive when at least one typical variety of labelling was observed in the frontal isocortex and/or the cerebellar vermis of the patient. In the study of tonsil biopsies, only patients with five or more secondary lymphoid follicles were considered $^{22,23}$ for immunostaining. The presence of intense immunoreactivity within germinal centres was considered as a positive result.

\section{Evaluation of the 12F10 Automated Fast Procedure in a Series of 300 Brains and 47 Tonsil Biopsies from Patients with a Suspected Diagnosis of Human Prion Diseases}

Between January 2001 and January 2006, 300 brains and 47 tonsil biopsies of patients with a suspected diagnosis of transmissible subacute encephalopathies were received at the R Escourolle Neuropathology Laboratory. PrP immunohistochemistry using the 12F10 automated fast procedure was performed on samples from frontal isocortex and cerebellar vermis, and tonsil biopsies. The slides were checked by two investigators (JJH and $\mathrm{SH}$ ), who were
Table 4 Antibodies screening with automation

\begin{tabular}{lccc}
\hline \multirow{2}{*}{ Antibodies } & \multicolumn{3}{c}{ Number of positive patients/total } \\
\cline { 2 - 4 } & All CJD forms & AD & NND \\
\hline 3F4 (m.p.) & $20 / 21(95.2 \%)$ & $0 / 3$ & $0 / 2$ \\
3F4 & $15 / 21(71.4 \%)$ & $0 / 3$ & $0 / 2$ \\
12F10 & $20 / 21(95.2 \%)$ & $0 / 3$ & $0 / 2$ \\
3B5 & $18 / 21(85.7 \%)$ & $3 / 3$ & $1 / 2^{*}$ \\
SAF34 & $18 / 21(85.7 \%)$ & $3 / 3$ & $1 / 2^{*}$ \\
BAR233 & $10 / 21(47.6 \%)$ & $0 / 3$ & $0 / 2$ \\
4F2 & $19 / 21(90.5 \%)$ & $3 / 3$ & $1 / 2^{*}$ \\
SAF54 & $19 / 21(90.5 \%)$ & $3 / 3$ & $1 / 2^{*}$ \\
\hline
\end{tabular}

AD, Alzheimer disease; CJD, Creutzfeldt-Jakob disease; NND, patients who died from non-neurological diseases; m.p., manual procedures (reference antibody).

${ }^{\star}$ Controls showing nonspecific background in the neuropile.

unaware of the diagnosis. Interobserver agreement for the diagnosis of transmissible subacute encephalopathies was higher than 0.95. A consensus was reached by joint examination of the remaining samples. The gold standard was the neuropathological examination of various brain areas using standard staining procedures coupled with the western blot demonstration of $\mathrm{PrP}^{\mathrm{sc}}$.

\section{Results}

\section{Antibodies Screening}

Step 1

When the 50 antibodies were screened using the $3 \mathrm{~F} 4$ reference procedure that includes four pretreatments, we found a strong immunoreactivity with three antibodies that had been produced using human recombinant PrP (4F2, 3B5, 12F10), two produced using denatured scrapie-associated fibrils from 263K-infected Syrian hamsters (SAF34, SAF54) and one produced using ovine recombinant PrP (BAR233) (Table 3). Working concentrations varied between 0.8 and $2 \mu \mathrm{g} / \mathrm{ml}$.

\section{Step 2}

When only one pretreatment (hydrated autoclaving) was used, a strong immunoreactivity was preserved 


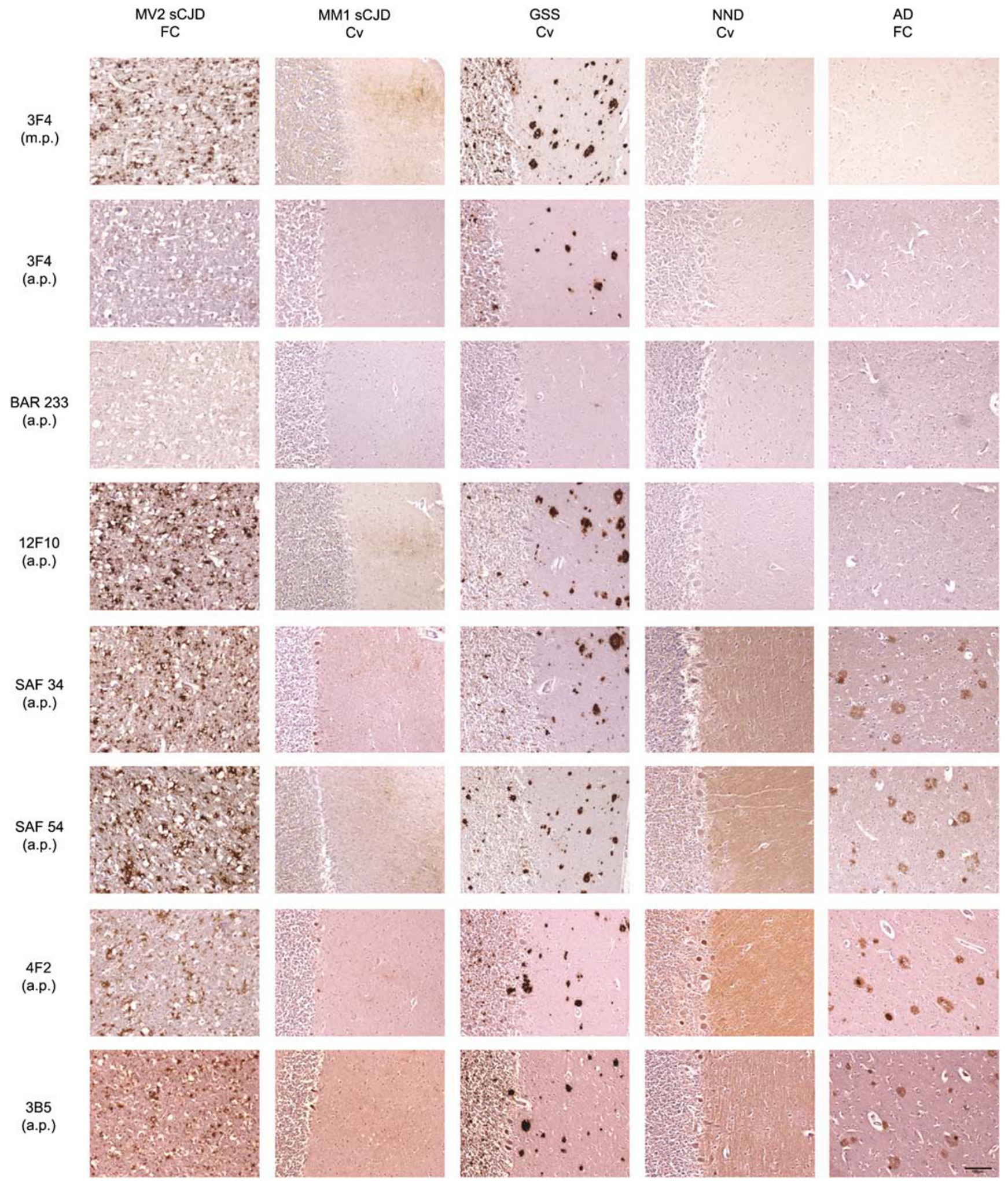

Figure 2 Immunostaining obtained with reference antibody 3F4 using manual procedure compared to 3F4, BAR233, 12F10, SAF34, SAf54, 4F2 and 3B5 antibodies using automated procedure. AD, Alzheimer disease patient; a.p., automated procedure; Cv, cerebellar vermis; FC, frontal isocortex; GSS, Gerstmann-Sträussler-Scheinker syndrome with the P102L mutation; MM1, methionine homozygosity at codon 129 of PRNP and a PrPsc type 1; m.p., manual procedure; MV2, methionine/valine heterozygosity at codon 129 and a PrP ${ }^{\mathrm{sc}}$ type 2A; NND, patient dead from a non-neurological disease; sCJD, sporadic Creutzfeldt-Jakob disease. Scale bar $=100 \mu \mathrm{m}$. 
for the six antibodies selected in step 1 (Table 3). As expected, when the number of pretreatments was reduced, the staining obtained using $3 \mathrm{~F} 4$ antibody dramatically decreased.

\section{Step 3}

The 12F10, 3B5, 4F2, SAF34 and SAF54 antibodies used in automated procedure provided a strong immunoreactivity, regardless of the pattern of $\mathrm{PrP}$ deposits. The 3F4 reference antibody did not work efficiently in the Nexes automated system $(71.4 \%$ sensitivity) (Table 4, Figure 2). Only the fatal familial insomnia (FFI) patient yielded negative results. There were two false-negative patients using 4F2 and SAF54 antibodies (90.5\% sensitivity) and three false-negative patients using 3B5 and SAF34 antibodies ( $85.7 \%$ sensitivity). BAR233 showed the lowest sensitivity $(47.6 \%)$. The 3B5, SAF34, 4F2 and SAF54 antibodies showed background on the neuropile and positive staining on the senile plaques of the three Alzheimer disease (AD) patients tested (Figure 2). When used in the automated system, 12 F10 was the most efficient antibody: sensitivity (95.5\%) and deposit patterns were similar to those of manual procedure of the $3 \mathrm{~F} 4$ reference antibody (Table 4, Figures 2 and 3).

\section{Step 4}

We compared the results of $3 \mathrm{~F} 4$ manual procedure to the 12F10 automated one using 51 patients (Table 5 , Figure 3). The sensitivity of the $12 \mathrm{~F} 10$ procedure was slightly higher than that of the 3F4 (95.7 vs $91.3 \%)$. In this step, we observed the characteristic patterns of the various studied transmissible subacute encephalopathies forms, regardless of the immunostaining procedure (Figure 3).

Evaluation of the 12F10 Automated Fast Procedure in a Series of 300 Patients with a Suspected Diagnosis of Human Transmissible Subacute Encephalopathies

To further validate the use of $12 \mathrm{~F} 10$ antibody in an automated procedure for the diagnosis of human transmissible subacute encephalopathies, we tested tissue sections from 300 brains of patients with suspected transmissible subacute encephalopathies. Neuropathological examination and western blot demonstration of proteinase K-resistant PrP confirmed the diagnosis of transmissible subacute encephalopathies in 169 patients out of the 300 suspected patients. Among these 169 patients, 161 were found positive in the frontal isocortex $(n=142)$, the cerebellar vermis $(n=159)$ or in both samples $(n=140)$. Only eight patients were negative in the two examined areas $(95.3 \%$ sensitivity) (Table 6). The 12 F10 automated procedure was efficient for the detection of $\mathrm{PrP}^{\mathrm{sc}}$ deposits in all forms of studied transmissible subacute encephalopathies: sporadic Creutzfeldt-Jakob disease (sCJD) $(n=147)$, iatrogenic CJD (iCJD) after extractive growth hormone treatment
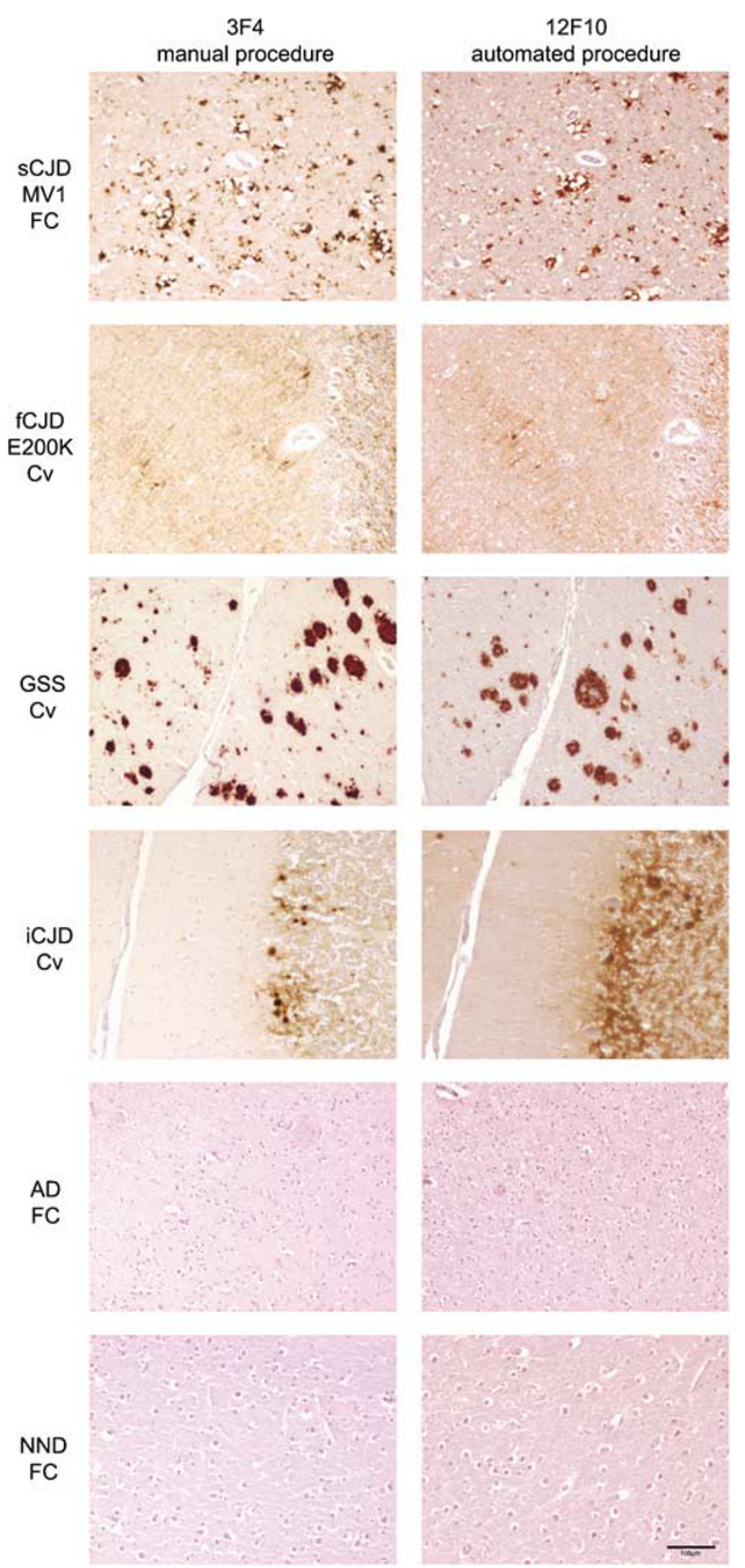

Figure 3 Immunostaining obtained with reference 3F4 antibody using a manual procedure compared to $12 \mathrm{~F} 10$ antibody using automated procedure. AD, Alzheimer disease; Cv, cerebellar vermis; fCJD E200K, familial Creutzfeldt-Jakob disease with the E200K mutation; FC, frontal isocortex; iCJD, iatrogenic Creutzfeldt-Jakob disease after extractive growth hormone treatment; MV1, methionine/valine heterozygosity at codon 129 and a PrP ${ }^{\text {sc }}$ type 1; NND, patient dead from a non-neurological disease; sCJD, sporadic Creutzfeldt-Jakob disease. Scale bar $=100 \mu \mathrm{m}$.

$(n=5)$, variant CJD (vCJD) $(n=3)$, and familial CJD (fCJD) $(n=14)$ with various mutations $(n=10)$. Only one out of the 131 patients with non-prion diseases was immunopositive (99.2\% specificity). 
Evaluation of the 12F10 Automated Fast Procedure in a Series of 47 Tonsil Biopsies from Patients with a Suspected Diagnosis of vCJD

To further validate the use of $12 \mathrm{~F} 10$ antibody in an automated procedure for the diagnosis of vCJD, we tested tissue sections from 47 tonsils of patients with suspected vCJD. Among these 47 patients, the final diagnosis was definite vCJD in 7 patients, probable vCJD in 2 and alternative diagnosis in 38. The 12F10 automated fast procedure showed positive results in all patients with definite or probable vCJD (Table 6). No positive staining was observed in the tonsils of patients with an alternative diagnosis. These results matched perfectly those of western blot assay after proteinase $\mathrm{K}$ digestion performed on tonsil samples from each patient.

\section{Discussion}

Identification of $\mathrm{PrP}^{\mathrm{sc}}$ in the central nervous tissue by $\mathrm{PrP}$ immunohistochemistry is a widely used technique for the diagnosis of human transmissible subacute encephalopathies. In this study, we compared 51 monoclonal antibodies (including the gold

Table 5 Validation of 12F10 automated procedure on a series of 51 patients and comparison with $3 \mathrm{~F} 4$ reference manual procedure

\begin{tabular}{lcc}
\hline \multirow{2}{*}{ Patients } & \multicolumn{2}{c}{ Number of positive patients/total } \\
\cline { 2 - 3 } & $\begin{array}{c}\text { 3F4 manual } \\
\text { procedure }\end{array}$ & $\begin{array}{c}12 F 10 \text { automated } \\
\text { procedure }\end{array}$ \\
\hline sCJD MM1 & $17 / 20$ & $19 / 20$ \\
sCJD MV1 & $8 / 8$ & $8 / 8$ \\
sCJD MV2 & $3 / 3$ & $3 / 3$ \\
sCJD VV2 & $6 / 6$ & $6 / 6$ \\
vCJD & $3 / 3$ & $3 / 3$ \\
fCJD E200K & $1 / 1$ & $1 / 1$ \\
GSS P102L & $1 / 1$ & $1 / 1$ \\
FFI D178N-129M & $0 / 1$ & $0 / 1$ \\
iCJD & $3 / 3$ & $3 / 3$ \\
AD & $0 / 3$ & $0 / 3$ \\
NND & $0 / 2$ & $0 / 2$ \\
All CJD forms & $42 / 46$ & $44 / 46$ \\
& $91.30 \%$ & $95.70 \%$ \\
\hline
\end{tabular}

AD, Alzheimer disease patient; iCJD, iatrogenic CJD after extractive growth hormone treatment; fCJD, inherited Creutzfeldt-Jakob disease with the E200K mutation; FFI, familial fatal insomnia with the D178N-129M mutation; MM1, methionine homozygosity at codon 129 of PRNP and a PrP ${ }^{\text {sc }}$ type 1; GSS, Gerstmann-Sträussler-Scheinker syndrome with the P102L mutation; MV1, methionine/valine heterozygosity at codon 129 and a $\operatorname{PrP}^{\text {sc }}$ type 1; MV2, methionine/valine heterozygosity at codon 129 and a PrPsc type 2A; NND, patients dead from non-neurological diseases; VV2, valine homozygosity at the codon 129 and a PrP ${ }^{\text {sc }}$ type 2A; sCJD, sporadic Creutzfeldt-Jakob disease; vCJD, variant Creutzfeldt-Jakob disease.

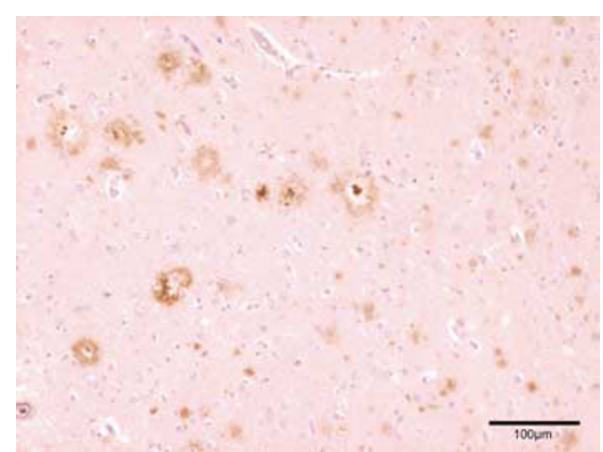

Figure 4 Immunostaining obtained with $12 \mathrm{~F} 10$ automated procedure showing positivities within $\beta$-amyloid deposits in the frontal isocortex, from a patient with AD. Scale bar $=100 \mu \mathrm{m}$.
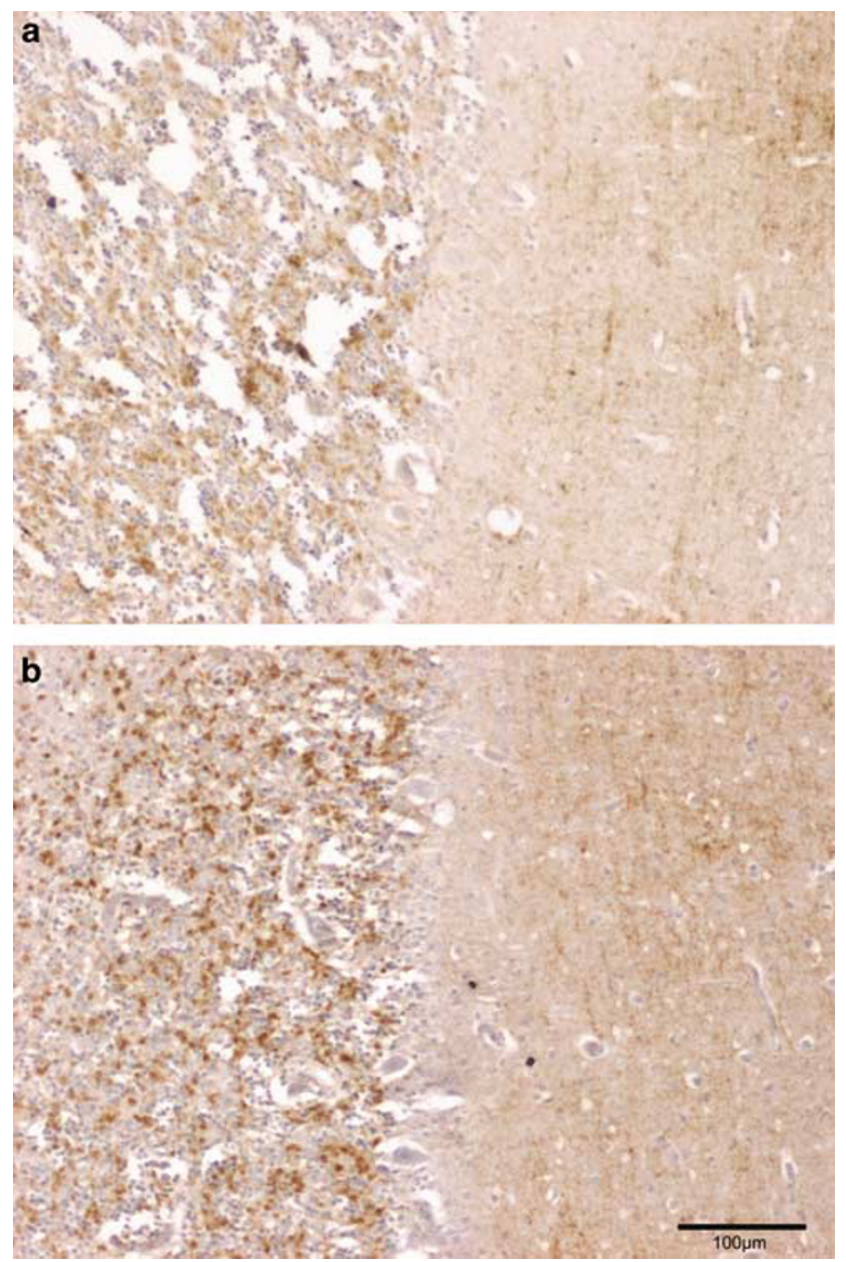

Figure 5 Tissue alterations induced by classical pretreatments used in 3F4 manual procedure (a) compared to only one pretreatment used in $12 \mathrm{~F} 10$ automated procedure (b) on serial sections of the cerebellar vermis from a patient with sporadic Creutzfeldt-Jakob disease. Scale bar $=100 \mu \mathrm{m}$.

Table 6 Evaluation of the 12 F10 automated procedures during a 5-year period in suspected CJD patients

Real positive Real negative False positive False negative Sensitivity in \% Specificity in \%

\begin{tabular}{|c|c|c|c|c|c|c|}
\hline PrP immunodetection on brain & 161 & 130 & 1 & 8 & 95.3 & 99.2 \\
\hline PrP immunodetection on tonsil biopsy & 9 & 38 & 0 & 0 & 100 & 100 \\
\hline
\end{tabular}


standard 3F4 antibody) and we developed a fast, reliable and automated procedure for PrP immunohistochemistry, allowing the diagnosis of the disease in more than $95 \%$ of the patients with a $99 \%$ specificity. Two brain areas were studied. The cerebellar vermis proved to be more often positive than the frontal isocortex.

It is worth noting that the epitopes of the antibodies that worked with limited pretreatment were located within the 59-161 region of the protein. The epitope 59-89 (4F2, 3B5 and SAF34 antibodies) and epitopes 144-152 (12F10), 157-161 (SAF54) and 141-151 (BAR233) flank the hydrophobic and potentially transmembrane domain of the protein (112-135).

In a previous study, Hainfellner et $a l^{24}$ have described an accumulation of $\mathrm{PrP}$ in senile plaques in some patients with $\mathrm{AD}$. We also observed a $\operatorname{PrP}$ immunostaining in $\beta$-amyloid deposits of $\mathrm{AD}$ patients using 4F2, 3B5, SAF34 and SAF54 antibodies (Figure 2). This probably corresponded to nonspecific labelling because (i) all these antibodies produced a strong nonspecific background in the neuropile, and (ii) all the studied AD patients were $\mathrm{PrP}$ positive using these antibodies. However, from our experience, $3 \mathrm{~F} 4$ and $12 \mathrm{~F} 10$ antibodies can also stain $\beta$-amyloid plaques in some $\mathrm{AD}$ patients (Figure 4). As previously reported, no $\mathrm{PrP}^{\text {sc }}$ was detected using western blot in these cases. The mechanics of co-aggregation of $\mathrm{PrP}^{\mathrm{c}}$ together with $\beta$-amyloid plaque compounds remains to be established.

Few monoclonal antibodies were effective when only one epitope-retrieving procedure (hydrated autoclaving) was used. This is less time-consuming and induces less tissue alterations, as illustrated in Figure 5. It also preserves epitopes of other proteins, which is beneficial for double immunostaining. The $12 \mathrm{~F} 10$ antibody could be used at $37^{\circ} \mathrm{C}$ in an automated procedure and provided the most sensitive and specific results. By comparison, the $12 \mathrm{~F} 10$ automated method was, in our hands, more sensitive than the other procedures using the reference 3F4 monoclonal antibody, which is not suitable for
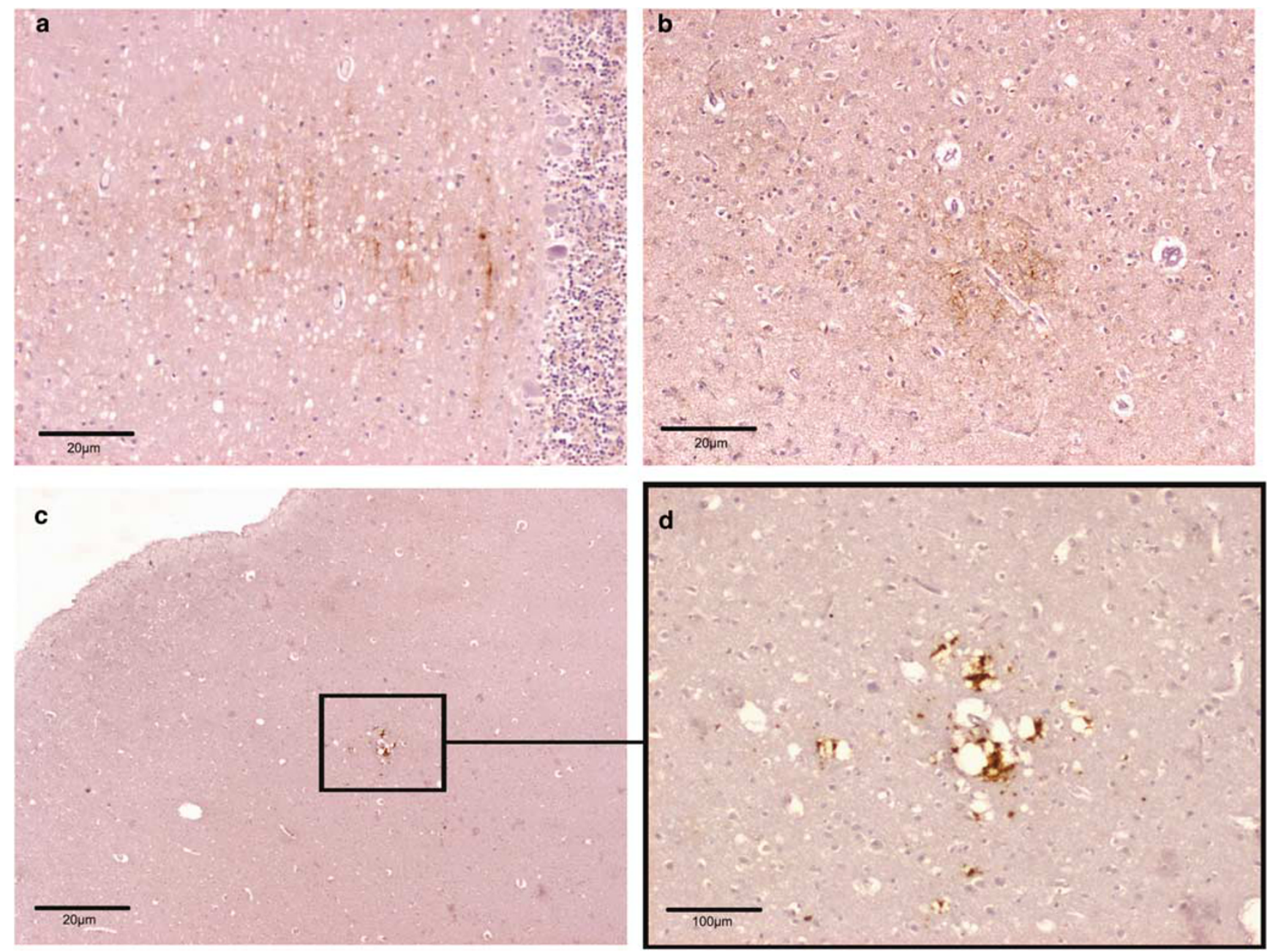

Figure 6 Immunostaining obtained with 12F10 automated procedure showing synaptic focal PrP deposits in the cerebellar vermis (a), frontal isocortex (b) and vacuolar focal deposits in frontal isocortex at different magnifications (c, d). Sections from a patient with sporadic Creutzfeldt-Jakob disease. 
this automated system. Our results confirm and extend the results of Kovacs et al, ${ }^{25}$ who identified $12 \mathrm{~F} 10$ as a potentially useful antibody for immunodiagnosis, owing to a reduction in the number of pretreatments and a speeding up of the procedure using a fast immunodiagnosis automate. In addition, we demonstrated the usefulness of this procedure in a very large panel of patients affected by a high diversity of human prion disorders.

It is worth noting that $\mathrm{PrP}$ immunostaining interpretation is sometimes difficult because $\mathrm{PrP}$ deposits may be very focal within the specimen. This is the case for synaptic deposits in the cerebellar or frontal cortex of some sCJD patients with methionine homozygosity and type $1 \mathrm{PrP}^{\mathrm{sc}}$ (Figure 6). Of the 169 patients with prion diseases, $12(7.1 \%)$ showed such focal deposits that may be difficult to identify for a pathologist who has not been trained in this technique of PrP immunohistochemistry.

This fast, automated procedure can be used for lymphoid tissue such as tonsil biopsy (nine tonsils from confirmed vCJD patients showed positive results consistently with the presence of type 2B $\mathrm{PrP}^{\mathrm{sc}}$ on western blot) (Figure 7).

Among 161 patients with transmissible subacute encephalopathies, only 8 showed negative immunohistochemical results (one patient with FFI, two fCJD patients associated with the D178N-129M or the V180I mutation and five patients with sCJD). The low level of $\mathrm{PrP}^{\mathrm{sc}}$ accumulation in FFI patients is a well-documented phenomenon. ${ }^{26}$ In all the eight transmissible subacute encephalopathy patients with negative immunohistochemical results, the western blot study yielded a positive result. Different methods have been developed, such as conformation-dependent immunoassay $(\mathrm{CDI})^{27}$ or protein misfolding cyclic amplification (PMCA), ${ }^{28,29}$ to increase the sensitivity of $\operatorname{PrP}^{\mathrm{sc}}$ detection. How these methods could improve routine diagnosis procedure in human pathology has to be confirmed using large case-control series. In terms of diagnosis of human transmissible subacute encephalopathies, developing highly sensitive methods such as CDI and PMCA may be more adapted to prion detection in peripheral tissue where very low $\mathrm{PrP}^{\mathrm{sc}}$ levels do occur (human blood). Considering our results using a fast $(2 \mathrm{~h})$, automated and highly sensitive procedure, more complex protocols would only slightly increase the sensitivity of detection. In addition, this procedure can be routinely applied in a general pathology laboratory.

In conclusion, after screening a large panel of antiPrP monoclonal antibodies, we developed a fast, reliable and automated procedure for $\mathrm{PrP}^{\mathrm{sc}}$ immunohistochemistry in human tissue sections. It allows a high sensitivity and specificity for the diagnosis of human prion diseases. The morphological information is preserved thanks to a simplified antigenretrieving procedure. This procedure was effective to detect $\mathrm{PrP}^{\mathrm{sc}}$ in the brain of almost all forms of
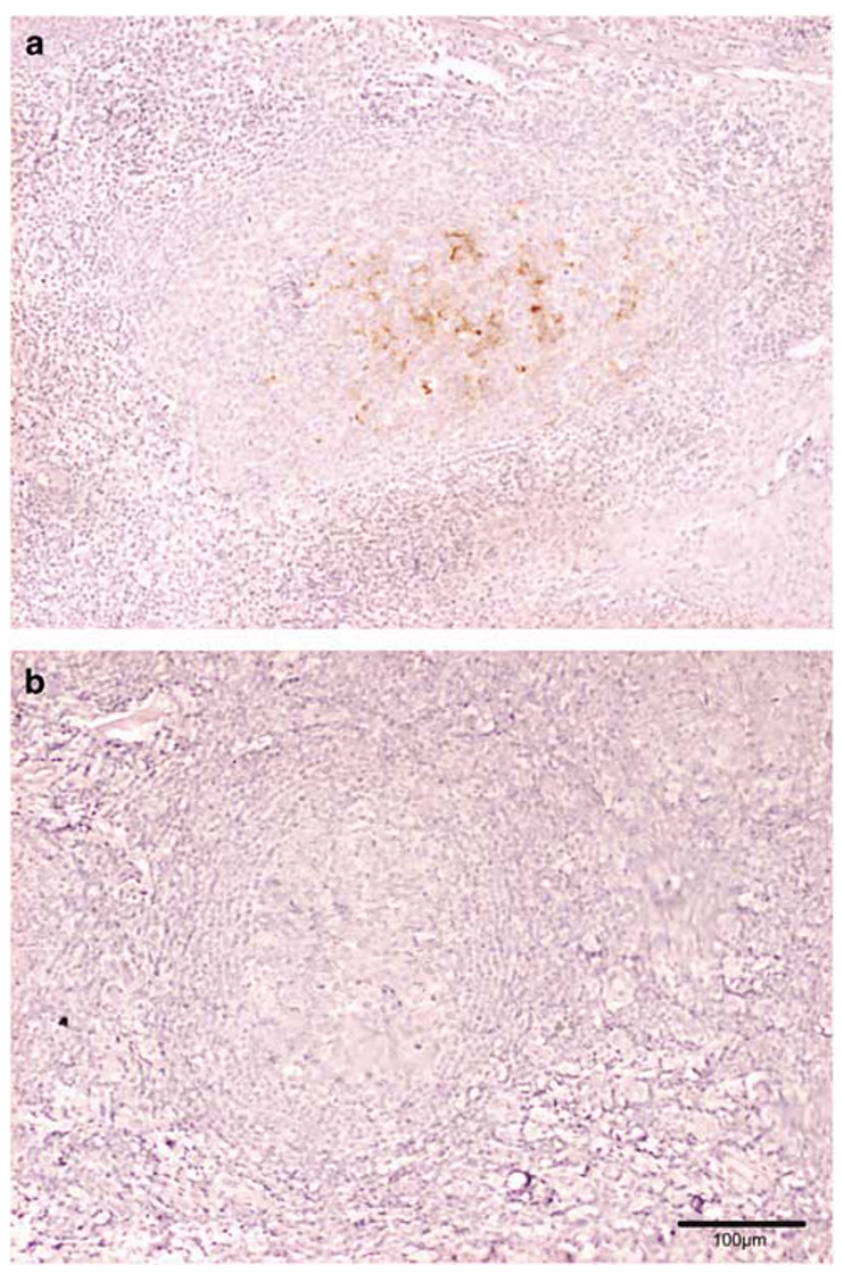

Figure 7 Immunostaining obtained with 12 F10 automated procedure in tonsil biopsy sections from a patient with variant Creutzfeldt-Jakob disease (a) and a patient who died from a non-neurological disease (b).

human transmissible subacute encephalopathies. It can be successfully applied in lymphoid tissue.

\section{Acknowledgements}

This work was supported in part by the GIS Prions and the EU Network of Excellence Neuroprion. We thank Agueda Matos and Emilie Morain for helpful technical assistance and Professor Gerhard Hunsmann (German Primate Centre, Goettingen, Germany) for hybridoma cells producing monoclonal antibodies directed against human recombinant $\mathrm{PrP}$ (4F2, 3B5, 12F10, 14D3, 8G8 and 11C6).

\section{References}

1 Ironside JW. Prion diseases in man. J Pathol 1998; 186:227-234.

2 Kretzschmar HA, Ironside JW, Dearmond SJ, et al. Diagnostic criteria for sporadic Creutzfeldt-Jakob disease. Arch Neurol 1996;53:913-920. 
3 Kitamoto T, Ogomori K, Tateishi J, et al. Formic acid pre-treatment enhances immunostaining of cerebral and systemic amyloids. Lab Invest 1987;57:230-236.

4 DeArmond SJ, Mobley WC, Demott DL, et al. Changes in the localization of brain prion proteins during scrapie infection. Neurology 1987;37:1271-1280.

5 Guiroy D, Yanagihara R, Gajdusek D. Localization of amyloidogenic proteins and sulfated glycosaminoglycans in nontransmissible and transmissible cerebral amyloidoses. Acta Neuropathol 1991;82:87-92.

6 Doi-Yi R, Kitamoto T, Tateishi J. Immunoreactivity of cerebral amyloidosis is enhanced by protein denaturation treatments. Acta Neuropathol 1991;82:260-265.

7 Kitamoto T, Shin RW, Dohura K, et al. Abnormal isoform of prion proteins accumulates in the synaptic structures of the central nervous system in patients with Creutzfeldt-Jakob disease. Am J Pathol 1992;140: 1285-1294.

8 Hashimoto K, Mannen T, Nukina N. Immunohistochemical study of kuru plaques using antibodies against synthetic prion protein peptides. Acta Neuropathol 1992;83:613-617.

9 Haritani M, Spencer YI, Wells GA. Hydrated autoclave pre-treatment enhancement of prion protein immunoreactivity in formalin-fixed bovine spongiform encephalopathy-affected brain. Acta Neuropathol (Berl) 1994;87:86-90.

10 Hayward P, Bell J, Ironside J. Prion protein immunocytochemistry: reliable protocols for the investigation of Creutzfeldt-Jakob disease. Neuropathol Appl Neurobiol 1994;20:375-383.

11 Macdonald ST, Sutherland K, Ironside JW. A quantitative and qualitative analysis of prion protein immunohistochemical staining in Creutzfeldt-Jakob disease using four anti prion protein antibodies. Neurodegeneration 1996;5:87-94.

12 Kascsak RJ, Rubenstein R, Merz PA, et al. Mouse polyclonal and monoclonal antibody to SAF (PrP) protein. J Virol 1987;61:3688-3693.

13 Bell JE, Gentleman SM, Ironside JW, et al. Prion protein immunocytochemistry-UK five centre consensus report. Neuropathol Appl Neurobiol 1997;23:26-35.

14 Privat N, Sazdovitch V, Seilhean D, et al. PrP immunohistochemistry: different protocols, including a procedure for long formalin fixation, and a proposed schematic classification for deposits in sporadic Creutzfeldt-Jakob disease. Microsc Res Tech 2000;50: 26-31.

15 Feraudet C, Morel N, Simon S, et al. Screening of 145 anti-PrP monoclonal antibodies for their capacity to inhibit PrPSc replication in infected cells. J Biol Chem 2005;280:11247-11258.
16 Morel N, Simon S, Frobert Y, et al. Selective and efficient immunoprecipitation of the disease-associated form of the prion protein can be mediated by nonspecific interactions between monoclonal antibodies and scrapie-associated fibrils. J Biol Chem 2004;279:30143-30149.

17 Reik LM, Maines SL, Ryan DE, et al. A simple, nonchromatographic purification procedure for monoclonal antibodies. Isolation of monoclonal antibodies against cytochrome $P 450$ isozymes. J Immunol Methods 1987;100:123-130.

18 Budka H, Aguzzi A, Brown P, et al. Neuropathological diagnostic criteria for Creutzfeldt-Jakob disease (CJD) and other human spongiform encephalopathies (prion diseases). Brain Pathol 1995;5:459-466.

19 Parchi P, Castellani R, Capellari S, et al. Molecular basis of phenotypic variability in sporadic CreutzfeldtJakob disease. Ann Neurol 1996;39:767-778.

20 Schulz-Schaeffer WJ, Giese A, Windl O, et al. Polymorphism at codon 129 of the prion protein gene determines cerebellar pathology in Creutzfeldt-Jakob disease. Clin Neuropathol 1996;15:353-357.

21 Hauw JJ, Sazdovitch V, Laplanche JL, et al. Neuropathologic variants of sporadic Creutzfeldt-Jakob disease and codon 129 of $\operatorname{PrP}$ gene. Neurology 2000; 54:1641-1646.

22 Hilton DA, Fathers E, Edwards $\mathrm{P}$, et al. Prion immunoreactivity in appendix before clinical onset of variant Creutzfeldt-Jakob disease. Lancet 1998;352: 703-704.

23 Hilton DA, Ghani AC, Conyers L, et al. Prevalence of lymphoreticular prion protein accumulation in UK tissue samples. J Pathol 2004;203:733-739.

24 Hainfellner J, Wanschitz J, Jellinger $\mathrm{K}$, et al. Coexistence of Alzheimer-type neuropathology in Creutzfeldt-Jakob disease. Acta Neuropathol 1998;96: 116-122.

25 Kovacs GG, Head MW, Hegyi I, et al. Immunohistochemistry for the prion protein: comparison of different monoclonal antibodies in human prion disease subtypes. Brain Pathol 2002;12:1-11.

26 Montagna P, Gambetti P, Cortelli P, et al. Familial and sporadic fatal insomnia. Lancet Neurol 2003;2: 167-176.

27 Safar JG, Geschwind MD, Deering C, et al. Diagnosis of human prion disease. Proc Natl Acad Sci USA 2005;102:3501-3506.

28 Castilla J, Saa P, Soto C. Detection of prions in blood. Nat Med 2005;11:982-985.

29 Saborio GP, Permanne B, Soto C. Sensitive detection of pathological prion protein by cyclic amplification of protein misfolding. Nature 2001;411:810-813. 